\title{
Cutis Verticis Gyrata Improved with Injected Hyaluronidase Treatments
}

\author{
Macartney Welborn ${ }^{1 *}$, Christopher Dallo ${ }^{2}$ and Mary Altmeyer ${ }^{3}$ \\ ${ }^{1}$ University of Texas McGovern Medical School, USA \\ ${ }^{2}$ University of Texas Medical Branch at Galveston, USA \\ ${ }^{3}$ Department of Dermatology, University of Texas at Health Science Center San Antonio, USA
}

Keywords

Cutis verticis gyrate, Hyaluronidase injections, Secondary cutis verticis gyrata

\section{Introduction}

Cutis verticis gyrata (CVG) is a condition of excessive skin growth on the scalp leading to deep furrows and folds that resemble the gyri of the brain. There are three main categories of CVG: primary essential, primary nonessential and secondary. Primary essential is idiopathic and presents only with cutaneous alterations [1]. Primary nonessential has cutaneous findings that can be associated with neurological or opthalmoglical pathology [2,3]. Primary CVG occurs more commonly in males and often develops before age 30 [1]. Secondary CVG are much more frequent and often a consequence of conditions that produce pathological changes in the scalp structure [4]. Causes of secondary CVG include severe local disorders, such as eczema and psoriasis, or systemic disorders such as diabetes mellitus [4]. We present a case of secondary CVG in a patient with longstanding underlying fibromatosis who presented with CVG two years into his disease. Standard of care for secondary CVG amelioration most commonly involves addressing the underlying systemic condition. Our patient, however, developed CVG while on systemic treatment for fibromatosis and had exceptional improvement with a course of local intralesional hyaluronidase injections. This is the first case report of hyaluronidase injections as a treatment option for CVG.

\section{Case Presentation}

Herein we present a case of a 47-year-old male with a past medical history significant for aggressive extra-abdominal fibromatosis of the chest wall referred to dermatology for new scalp lesions. Two years prior to presentation, a left chest mass was diagnosed and resected. One year later, he felt pain and a mass in the previously treated area. MRI of the chest revealed three recurrent masses and enhancing left axillary nodes. He underwent resection of the tumor and was started on $60 \mathrm{mg}$ Tamoxifen, $300 \mathrm{mg}$ Sulindac and $400 \mathrm{mg}$ of Imatinib. The patient's disease improved after treatment and is currently stable. A year post treatment initiation scalp lesions developed. Physical examination demonstrated multiple symmetric ridges and grooves in the scalp, most prominent over the vertex and occiput (Figure 1). A punch biopsy of the scalp showed focal alopecia, dermal fibrosis, and edema consistent with cutis verticis gyrata. He was treated with 150 units of hyaluronidase injections six weeks apart for six total treatments. Improvement was seen after three treatments with the with the hyaluronidase injections (Figure 2 and Figure 3). The furrows were less prominent throughout the scalp and there were areas of hair growth throughout the scalp. The patient tolerated the treatment well with no untoward side effects.

\section{Discussion}

Cutis verticis gyrata (CVG) is a condition of excessive skin growth on the scalp leading to deep furrows and folds that resemble the gyri of the brain. The area's most commonly involved include the vertex and occipital scalp. The condition tends to be most bothersome for patients cosmetically. Patients, however, can also develop accumulation of material in the grooves leading to a disturbing odor, burning sensation, pruritus and irritation of the area [5]. Standard of care for treating CVG includes surgical intervention for cosmetic

*Corresponding author: Macartney Welborn B.S., University of Texas McGovern Medical School, 1515 Holcombe Blvd, FCT 11.6078, Unit 1452, Houston, Texas 77030-4009, USA, Tel: 210289-6906, Fax: 713-745-3597

Accepted: January 03, 2019

Published online: January 05, 2019

Citation: Welborn M, Dallo C, Altmeyer M (2018) Cutis Verticis Gyrata Improved with Injected Hyaluronidase Treatments. Dermatol Arch 3(1):74-76 


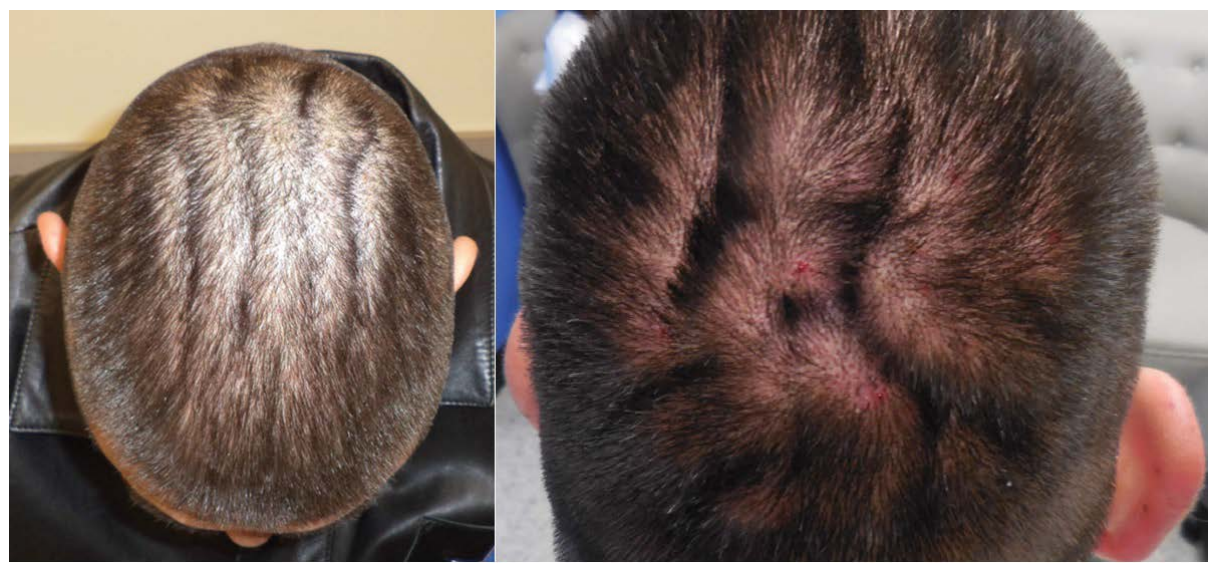

Figure 1: Multiple symmetric ridges and grooves in the vertex (left) and occiput (right) scalp.

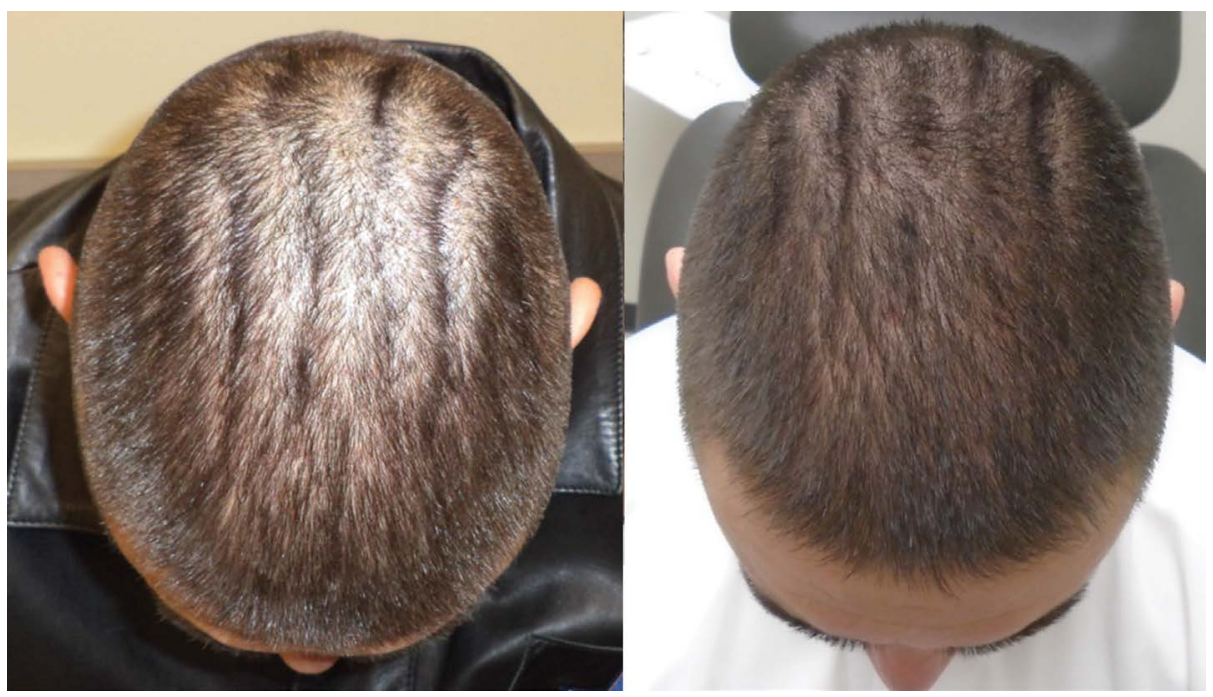

Figure 2: Improvement of ridges and grooves in vertex scalp at week 0 of treatment (left) and week 18 of treatment (right).

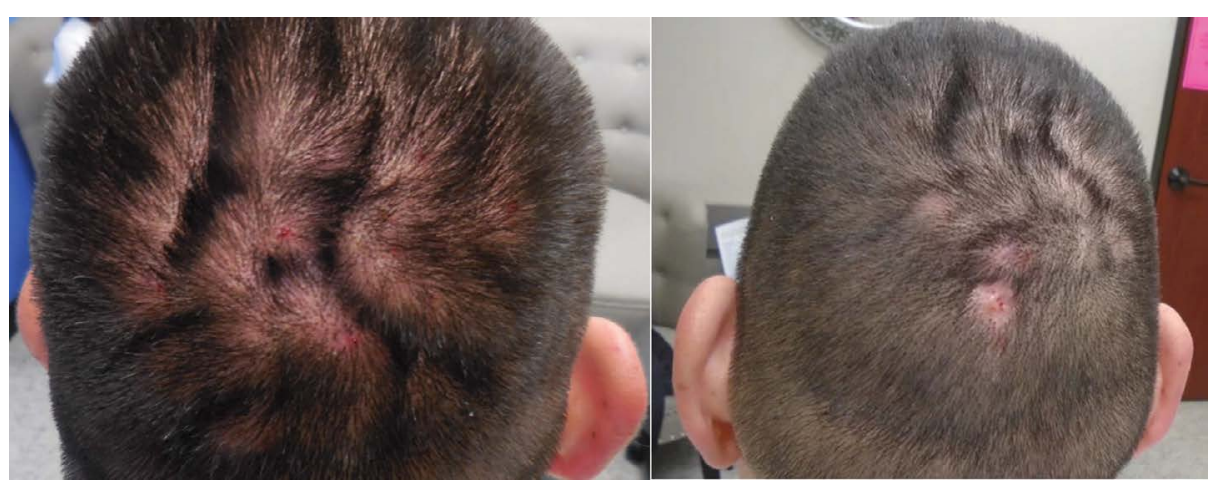

Figure 3: Improvement of ridges and grooves in occiput scalp at week 0 of treatment (left) and week 18 of treatment (right).

purposes and, in the setting of secondary CVG, treatment is aimed at addressing the underlying systemic disease. This is the first report of a case with an alternative treatment regimen using hyaluronidase injection, a potentially less invasive treatment option for CVG which has a low side effect profile.

This patient was treated with six injections of 150 units of hyaluronidase every six weeks. After three weeks, the patient started to show improvement. Hyaluronidase is an enzyme that catalyzes the degradation of hyaluronic acid which is an essential component found in the extracellular matrix of skin [6]. Hyaluronic acid is a linear glycosaminoglycan disaccharide that is found attached to proteoglycans and is hydrophilic which allows it to contribute to the elastic properties of the skin [7]. In fact, the most common glycosaminoglycan found in the dermis is hyaluronic acid [8]. Hyaluronidase is used in European countries as a local adjuvant to increase the diffusion capacity of local anesthetics. There is increasing 
off-label use of hyaluronidase for hyaluronic acid -fillerassociated complications. Loss of dermal hyaluronic acid is a hallmark of skin aging and the use of hyaluronic acidbased fillers has become a treatment of choice in tissue augmentation [9]. Although the exact mechanism of how hyaluronidase improved this patient's CVG is unknown, it can be theorized that by decreasing hyaluronic acid in the area where skin overgrowth had occurred can lead to decreased skin elasticity in those areas which was the desired cosmetic effect for this condition.

As treatment options are limited for CVG and often require invasive measures, hyaluronidase injections provide an interesting alternative that can greatly improve patient's quality of life. We describe the first reported case of hyaluronidase injections improving the cutaneous manifestations of CVG. As this is only one case, no definite conclusion can be drawn but it does raise the idea for further prospective research to establish if these findings can be repeated for other CVG cases.

\section{References}

1. Ennouhi MA, Guerrouani A, Moussaoui A (2018) Idiopathic cutis verticis gyrata in a female. Cureus 10: e2105.

2. Tan O, Ergen D (2006) Primary essential cutis verticis gyrata in an adult female patient: A case report. J Dermatol 33: 492-495.

3. Tucci A, Pezzani L, Scuvera G, et al. (2017) Is cutis verticis gyrataintellectual disability syndrome an underdiagnosed condition? A case report and review of 62 cases. Am J Med Genet A 173: 638-646.

4. Nguyen NQ (2003) Cutis verticis gyrata. Dermatol Online J 9: 32.

5. Yagerman S, Callahan S, Terushkin V, et al. (2016) Cutis verticis gyrata. Dermatol Online J 22.

6. Juhlin L (1997) Hyaluronan in skin. J Intern Med 242: 61-66.

7. Laurent TC, Fraser JR (1992) Hyaluronan. FASEB J 6: 2397-2404.

8. Stern R, Maibach HI (2008) Hyaluronan in skin: Aspects of aging and its pharmacologic modulation. Clin Dermatol 26: 106-122.

9. Hirsch RJ, Brody HJ, Carruthers JD (2007) Hyaluronidase in the office: A necessity for every dermasurgeon that injects hyaluronic acid. J Cosmet Laser Ther 9: 182-185. 